\title{
On the control of the lateral Casimir force between corrugated surfaces
}

\author{
E. V. Blagov, ${ }^{1}$ G. L. Klimchitskaya, ${ }^{2}$ U. Mohideen,${ }^{3}$ and V. M. Mostepanenko ${ }^{1,2}$ \\ ${ }^{1}$ Noncommercial Partnership "Scientific Instruments", Moscow, Russia \\ ${ }^{2}$ Departamento de Física, Universidade Federal da Paraíba, \\ C.P.5008, CEP 58059-970, João Pessoa, Pb-Brazil \\ ${ }^{3}$ Department of Physics, University of California, Riverside, California 92521
}

\begin{abstract}
The general perturbative expression for the lateral Casimir force between two plates covered by longitudinal corrugations of arbitrary shape is obtained. This expression is applicable for corrugation periods larger than the separation distance. The cases of asymmetric corrugations are considered, which allow to increase the maximum to minimum force ratio and affect the character of equilibrium points. This opens new opportunities to control the lateral Casimir forces for use in microelectromechanical devices based entirely on the vacuum fluctuation properties.
\end{abstract}

PACS numbers: $12.20 . \mathrm{Ds}, 42.50 . \mathrm{Lc}$ 
It is well known that the Casimir force 1] arises due to electromagnetic quantum fluctuations and acts between closely spaced surfaces. For short distances of order a nanometer, the limiting form of the Casimir force results in the van der Waals force. In fact the Casimir force is a collective quantum phenomenon which results from the alteration of the zero-point photon spectrum by surfaces of material bodies. H. B. G. Casimir [1] was the first to calculate an extraordinary property that two parallel uncharged metallic plates placed in vacuum at some separation $a$ would feel an attractive force per unit area $F_{0}(a)=-\pi^{2} \hbar c /\left(240 a^{4}\right)$, where $\hbar$ is the Planck constant and $c$ is the velocity of light. The Casimir force exhibits important yet exotic dependencies on the shape of boundary surfaces, which are not simple extensions from flat boundaries (see monographs [2, 3, 4] and reviews [5, 6]).

Recently a number of experiments were performed on measuring the normal Casimir force, acting perpendicular to the interacting surfaces [7, 8, 9, 10, 11, 12, 13, 14, 15]. The highest precision of order of $1 \%$ of the measured force was achieved in the measurements by means of atomic force microscope [8, 9, 10, 11] and microelectromechanical torsional oscillator [14, 15]. The extent of agreement between experiment and theory was used to set stringent limits on predictions of fundamental physical theories [15, 16, 17, 18, 19]. The Casimir force can be the dominant force acting between surfaces for separation distances on the order or less than a few hundred nanometers. The Casimir effect was used to produce mechanical motion of microfabricated silicon plate [20]. This is the first case of microelectromechanical device which shows actuation by the Casimir force. Note also that the Casimir forces acting between closely spaced surfaces play the crucial role in the fabrication and yield of microdevices due to the phenomena of adhesion and stiction [21].

Similar to the normal Casimir force, the lateral Casimir force originates from the modifications of zero-point oscillations by material boundaries. It acts tangential to the two surfaces with nanoscale periodic corrugations. To first order the origin of the lateral Casimir force can be simply understood as follows. As the Casimir force is a strong function of distance $\left(F_{0} \sim a^{-4}\right)$, the natural tendency is for corrugated plates to align themselves such that peaks of the top plate are directly over the peaks of the bottom plate. This force to align the peaks is then the origin of the lateral force acting in the horizontal direction between the plates. The lateral Casimir force for anisotropic boundaries was investigated theoretically and a harmonic dependence on corresponding angle was predicted [3, 22, 23]. For two aligned sinusoidally corrugated plates made of ideal metal the lateral Casimir force was discussed 
in Refs. [24, 25] and a harmonic dependence on a phase shift between corrugations was found. In Ref. [26] the first experimental observation of the lateral Casimir force between the sinusoidally corrugated plate and large corrugated sphere was reported. The predicted sinusoidal dependence on the phase shift between corrugations was confirmed. The analysis of the theoretical dependence for the lateral Casimir force was performed in Ref. [26] and the optimum values of the parameters providing the maximum values of the lateral force in the case of sinusoidal corrugations were found.

In this report we investigate the lateral Casimir force between two parallel plates covered by longitudinal grooves of arbitrary shape. This opens new opportunities to change the magnitude of the lateral Casimir force and obtain asymmetric lateral forces with a more complicated character of equilibrium points leading to a rich variety of surface interactions. As a consequence, it may become possible to control friction through nanoscale modification of the contact surfaces to increase the yield of microdevices [21].

The normal Casimir force acting between plates covered with small distortions has been the subject of much attention in literature (see Ref. 6] for review). In Ref. [27] a general method was provided which enables one to find corrections to the normal Casimir force in the configuration of two parallel plates with small deviations from plane parallel geometry. This method is based on the pairwise summation of the interatomic Casimir potentials with a subsequent normalization of the obtained interaction constant [3, 6]. In doing so, the amplitudes of deviations from plane parallel geometry $A_{1,2}$ are assumed to be small as compared to the separation $a$ between plates.

To fix the definition of the separation between the nonplanar plates, we describe their surfaces by the equations

$$
z_{1}^{(s)}=A_{1} f_{1}(x, y), \quad z_{2}^{(s)}=a+A_{2} f_{2}(x, y)
$$

where $a$ is the mean value of the distance between the square plates with a side $2 L$ and the coordinate system is connected with the lower plate. The values of the amplitudes are chosen in such a way that $\max \left|f_{i}(x, y)\right|=1$. The zero point in the $z$-axis is so chosen that

$$
\begin{aligned}
& \left\langle z_{1}^{(s)}\right\rangle \equiv A_{1}\left\langle f_{1}(x, y)\right\rangle=\frac{A_{1}}{4 L^{2}} \int_{-L}^{L} d x \int_{-L}^{L} d y f_{1}(x, y)=0, \\
& \left\langle z_{2}^{(s)}\right\rangle \equiv a+A_{2}\left\langle f_{2}(x, y)\right\rangle=a .
\end{aligned}
$$

The normal Casimir force between the plates covered with distortions was represented 
by a perturbative expansion up to the fourth order [27]

$$
F(a)=F_{0}(a) \sum_{k=0}^{4} \sum_{l=0}^{4-k} c_{k l}\left(\frac{A_{1}}{a}\right)^{k}\left(\frac{A_{2}}{a}\right)^{l},
$$

where $F_{0}(a)$ is the Casimir force per unit area of flat plates defined earlier, $c_{00}=1$ and $c_{01}=c_{10}=0$ [this follows from our choice (2)]. Explicit expressions for the other coefficients $c_{k l}$ are found in Ref. [27] (see also Refs. [3, 6]). They are rather combersome. If, however, both functions $f_{1}$ and $f_{2}$ are periodic in two variables with periods much larger than $a$, but much less than $2 L$, simple approximate expressions for $c_{k l}$ follow leading to

$$
\begin{aligned}
F(a) & =F_{0}(a)\left[1+\frac{10}{a^{2}}\left(\left\langle f_{1}^{2}\right\rangle A_{1}^{2}-2\left\langle f_{1} f_{2}\right\rangle A_{1} A_{2}+\left\langle f_{2}^{2}\right\rangle A_{2}^{2}\right)\right. \\
+ & \frac{20}{a^{3}}\left(\left\langle f_{1}^{3}\right\rangle A_{1}^{3}-3\left\langle f_{1}^{2} f_{2}\right\rangle A_{1}^{2} A_{2}+3\left\langle f_{1} f_{2}^{2}\right\rangle A_{1} A_{2}^{2}-\left\langle f_{2}^{3}\right\rangle A_{2}^{3}\right) \\
+ & \left.\frac{35}{a^{4}}\left(\left\langle f_{1}^{4}\right\rangle A_{1}^{4}-4\left\langle f_{1}^{3} f_{2}\right\rangle A_{1}^{3} A_{2}+6\left\langle f_{1}^{2} f_{2}^{2}\right\rangle A_{1}^{2} A_{2}^{2}-4\left\langle f_{1} f_{2}^{3}\right\rangle A_{1} A_{2}^{3}+\left\langle f_{2}^{4}\right\rangle A_{2}^{4}\right)\right] .
\end{aligned}
$$

Here the averaging as in Eq. (2) is done over the periods.

From Eq. (4) the Casimir energy per unit area can be found

$$
\begin{aligned}
E(a) & =\int_{a}^{\infty} F\left(a^{\prime}\right) d a^{\prime}=E_{0}(a)\left[1+\frac{6}{a^{2}}\left(\left\langle f_{1}^{2}\right\rangle A_{1}^{2}-2\left\langle f_{1} f_{2}\right\rangle A_{1} A_{2}+\left\langle f_{2}^{2}\right\rangle A_{2}^{2}\right)\right. \\
& +\frac{10}{a^{3}}\left(\left\langle f_{1}^{3}\right\rangle A_{1}^{3}-3\left\langle f_{1}^{2} f_{2}\right\rangle A_{1}^{2} A_{2}+3\left\langle f_{1} f_{2}^{2}\right\rangle A_{1} A_{2}^{2}-\left\langle f_{2}^{3}\right\rangle A_{2}^{3}\right) \\
& \left.+\frac{15}{a^{4}}\left(\left\langle f_{1}^{4}\right\rangle A_{1}^{4}-4\left\langle f_{1}^{3} f_{2}\right\rangle A_{1}^{3} A_{2}+6\left\langle f_{1}^{2} f_{2}^{2}\right\rangle A_{1}^{2} A_{2}^{2}-4\left\langle f_{1} f_{2}^{3}\right\rangle A_{1} A_{2}^{3}+\left\langle f_{2}^{4}\right\rangle A_{2}^{4}\right)\right],
\end{aligned}
$$

where $E_{0}(a)=-\pi^{2} \hbar c /\left(720 a^{3}\right)$ is the Casimir energy between two flat plates.

Now let functions $f_{1,2}$ depend on only one variable, say $x$, i.e. they describe longitudinal corrugations extending in the $y$ direction. Let their periods be equal, $\Lambda_{1}=\Lambda_{2}=\Lambda$, but there may exist some phase shift $x_{0}$. In this case $E(a)=E\left(a, x_{0}\right)$ and the lateral Casimir force arises directed along $x$-axis. It can be found from Eq. (5) as

$$
\begin{aligned}
& F^{l a t}\left(a, x_{0}\right)=-\frac{\partial E\left(a, x_{0}\right)}{\partial x_{0}}=F_{0}(a) \frac{2 A_{1} A_{2}}{a^{2}}\left[2 \frac{\partial}{\partial x_{0}}\left\langle f_{1} f_{2}\right\rangle\right. \\
& +5\left(\frac{A_{1}}{a} \frac{\partial}{\partial x_{0}}\left\langle f_{1}^{2} f_{2}\right\rangle-\frac{A_{2}}{a} \frac{\partial}{\partial x_{0}}\left\langle f_{1} f_{2}^{2}\right\rangle\right) \\
& \left.+10\left(\frac{A_{1}^{2}}{a^{2}} \frac{\partial}{\partial x_{0}}\left\langle f_{1}^{3} f_{2}\right\rangle-\frac{3}{2} \frac{A_{1} A_{2}}{a^{2}} \frac{\partial}{\partial x_{0}}\left\langle f_{1}^{2} f_{2}^{2}\right\rangle+\frac{A_{2}^{2}}{a^{2}} \frac{\partial}{\partial x_{0}}\left\langle f_{1} f_{2}^{3}\right\rangle\right)\right] .
\end{aligned}
$$

Eq. (6) permits to find the lateral Casimir force between plates covered by the longitudinal corrugations of any shape. Under the condition used $\Lambda \gg a$ the precision of the pairwise 
summation method is very high and the results practically coincide with the exact ones (this was demonstrated in Ref. [25] for the case of sinusoidal corrugations). The same conclusion can be obtained by the method of Ref. [28]. The advantage of Eq. (66) is the possibility to quickly obtain the results for corrugations of any shape and to generalize this formalism for the case of real metals of finite conductivity (see Ref. [26]).

As was shown in Ref. [26] for the sinusoidal corrugations, the dependence of the lateral force on the phase shift is almost sinusoidal. Specifically, the points of the stable and unstable equilibrium (where $F^{l a t}=0$ ) alternate with half the corrugation period. The magnitudes of the maximum and minimum values of $F^{l a t}$ are equal to each other so that their ratio is equal to unity. These characteristic features of the lateral Casimir force are preserved for any longitudinal corrugation whose shape is symmetric relatively to some vertical axis.

In applications to microelectromechanical systems, a more complicated nature of the lateral Casimir force may be desirable, i.e. different magnitudes of maximum and minimum values and a more complicated character of the points of equilibrium. This can be achieved by use of asymmetric longitudinal grooves on the plate surfaces. We consider saw toothed corrugations with equal amplitudes shown in Fig. 1,a. Within the periods (from 0 to $\Lambda$ for $f_{1}$ and from $x_{0}$ to $x_{0}+\Lambda$ for $f_{2}$ ) the analytical representations of the corrugation functions of Fig. 1,a are

$$
f_{1}(x)=\frac{2 x}{\Lambda}-1, \quad f_{2}(x)=1-\frac{2\left(x-x_{0}\right)}{\Lambda} .
$$

Calculating all matrix elements from Eq. (6) over the period from 0 to $\Lambda$, one obtains

$$
\begin{aligned}
& \left\langle f_{1} f_{2}\right\rangle=-\frac{1}{3}+2 \frac{x_{0}}{\Lambda}-2 \frac{x_{0}^{2}}{\Lambda^{2}} \\
& \left\langle f_{1}^{2} f_{2}\right\rangle=\left\langle f_{1} f_{2}^{2}\right\rangle=-\frac{4}{3} \frac{x_{0}}{\Lambda}+4 \frac{x_{0}^{2}}{\Lambda^{2}}-\frac{8}{3} \frac{x_{0}^{3}}{\Lambda^{3}}, \\
& \left\langle f_{1}^{3} f_{2}\right\rangle=\left\langle f_{1} f_{2}^{3}\right\rangle=-\frac{1}{5}+2 \frac{x_{0}}{\Lambda}-6 \frac{x_{0}^{2}}{\Lambda^{2}}+8 \frac{x_{0}^{3}}{\Lambda^{3}}-4 \frac{x_{0}^{4}}{\Lambda^{4}} \\
& \left\langle f_{1}^{2} f_{2}^{2}\right\rangle=\frac{1}{5}-\frac{8}{3} \frac{x_{0}^{2}}{\Lambda^{2}}+\frac{16}{3} \frac{x_{0}^{3}}{\Lambda^{3}}-\frac{8}{3} \frac{x_{0}^{4}}{\Lambda^{4}} .
\end{aligned}
$$

Substitution of Eq. (8) into Eq. (6) with $A_{1}=A_{2}=A$ leads to the result

$$
F^{l a t}\left(a, x_{0}\right)=8\left|F_{0}(a)\right| \frac{A^{2}}{a \Lambda}\left(2 \frac{x_{0}}{\Lambda}-1\right)\left[1+10 \frac{A^{2}}{a^{2}}\left(1-2 \frac{x_{0}}{\Lambda}+2 \frac{x_{0}^{2}}{\Lambda^{2}}\right)\right] \text {. }
$$

As an illustration, Fig. 2, a shows the dependence of $F^{\text {lat }} /\left|F_{0}\right|$ on $x_{0} / \Lambda$ computed by Eq. (9) with the typical values of parameters $A / a=0.3$ and $a / \Lambda=0.2$. From this figure 
it is seen that the lateral force is still symmetric and the points of unstable equilibrium $\left(x_{0} / \Lambda=0.5,1.5, \ldots\right)$ are exactly in the middle between the points of stable equilibrium $\left(x_{0} / \Lambda=0,1,2, \ldots\right)$. Like in the case of the sinusoidal corrugations, the lateral force is negative over one half of the period and positive over another half. The magnitudes of maximum and minimum values of the force are equal. At the same time, the case of saw toothed structures is different from sinusoidal corrugations because here the extremum values of the lateral force are achieved near the points of stable equilibrium (where the force is discontinuous). The points of stable equilibrium in this configuration are especially stable. Even small deviation from the stable equilibrium (where the value of the lateral force is taken equal to zero, i.e., half a sum of the limiting values from the left and from the right) leads to a large lateral force, restoring the state of equilibrium. Thus, the plates with saw toothed corrugations could be used in microdevices where the lateral displacements of the elements should be avoided (e.g. in devices such as micromirrors, microgears, micropumps, microsensors and microvalves).

Now we consider even more asymmetric longitudinal corrugations on the lower plate allowing to obtain different magnitudes for the maximum and minimum values of the lateral Casimir force. On the upper plate the same corrugations as in the previous example are preserved. The profiles are shown in Fig. 1,b. The new parameter $\Delta=l_{x} / \Lambda$ characterizes the extent of asymmetry. If $l_{x}=0(\Delta=0)$, the profiles in Fig. 1,b coincide with those in Fig. 1,a. The function $f_{1}$, describing the lower plate, can be presented as (within one period)

$$
f_{1}(x)=\left\{\begin{aligned}
-\frac{1-\Delta}{1+\Delta}, & 0<x \leq l_{x}, \\
\frac{2}{1-\Delta^{2}} \frac{x}{\Lambda}-\frac{1+\Delta^{2}}{1-\Delta^{2}}, & l_{x}<x \leq \Lambda .
\end{aligned}\right.
$$

The function $f_{2}$ for the upper plate is given by Eq. (17). Note that according to Eq. (2) both functions have zero mean values over the period.

The expression for the lateral Casimir force is obtained by calculating the matrix elements from Eq. (6) over the period $(0, \Lambda)$. Here the result takes a different form depending on whether $x_{0} \leq l_{x}$ or $x_{0} \geq l_{x}$. As before, we consider $A_{1}=A_{2}$. Then for $x_{0} \leq l_{x}$ one obtains

$$
\begin{gathered}
F^{l a t}\left(a, x_{0}\right)=-8\left|f_{0}(a)\right| \frac{A^{2}}{a \lambda} \frac{1-\Delta}{1+\Delta}\left\{1+\frac{10}{3} \frac{A}{a} \frac{1}{1+\Delta}\left[\Delta(3+\Delta)-3 \frac{x_{0}}{\Lambda}(1+\Delta)\right]\right. \\
\left.+10 \frac{A^{2}}{a^{2}}\left[\frac{1+5 \Delta^{2}+4 \Delta^{3}+\Delta^{4}}{(1+\Delta)^{2}}-4 \frac{x_{0}}{\Lambda} \frac{\Delta(3+\Delta)}{1+\Delta}+6 \frac{x_{0}^{2}}{\Lambda^{2}}\right]\right\} .
\end{gathered}
$$


For large phase shifts $x_{0} \geq l_{x}$ the expression for the lateral Casimir force is a bit more complicated

$$
F^{l a t}\left(a, x_{0}\right)=8\left|f_{0}(a)\right| \frac{A^{2}}{a \lambda} \frac{1}{1-\Delta^{2}}\left(2 \frac{x_{0}}{\Lambda}-1-\Delta^{2}\right)\left(1+\frac{10}{3} \frac{A}{a} X_{1}+10 \frac{A^{2}}{a^{2}} X_{2}\right),
$$

where the coefficients $X_{1,2}$ are given by

$$
\begin{aligned}
X_{1}= & -\frac{\Delta^{2}\left[2-3 \Delta+3 \Delta^{2}+\Delta^{3}-3\left(1+\Delta^{2}\right) x_{0} / \Lambda+3 x_{0}^{2} / \Lambda^{2}\right]}{\left(1-\Delta^{2}\right)\left(1+\Delta^{2}-2 x_{0} / \Lambda\right)}, \\
X_{2}= & \frac{1}{\left(1-\Delta^{2}\right)^{2}\left(1+\Delta^{2}-2 x_{0} / \Lambda\right)}\left[1-\Delta^{2}+10 \Delta^{4}-12 \Delta^{5}+\Delta^{6}\right. \\
& +4 \Delta^{7}+\Delta^{8}-4 \frac{x_{0}}{\Lambda}\left(1-\Delta^{2}+3 \Delta^{3}-4 \Delta^{5}+3 \Delta^{6}+\Delta^{7}\right) \\
& \left.+6 \frac{x_{0}^{2}}{\Lambda^{2}}\left(1+\Delta^{6}\right)-4 \frac{x_{0}^{3}}{\Lambda^{3}}\left(1-\Delta^{2}+\Delta^{4}\right)\right] .
\end{aligned}
$$

Direct calculation shows that for $x_{0}=l_{x}$ Eqs. (11) and (12) lead to one and the same result. Furthermore, for $\Delta=0$ one obtains $X_{1}=0, X_{2}=1-2\left(x_{0} / \Lambda\right)+2\left(x_{0} / \Lambda\right)^{2}$, so that Eq. (12) coincides with Eq. (9) as required (because the profiles in Figs. 1,a,b coincide when $\Delta=0$ ).

The coordinate of the point of unstable equilibrium, where $F^{l a t}\left(a, \tilde{x}_{0}\right)=0$, is found from Eq. (12)

$$
\tilde{x}_{0}=\frac{\Lambda\left(1+\Delta^{2}\right)}{2}
$$

For example, in Fig. 2,b one period of the relative lateral Casimir force $F^{l a t} /\left|F_{0}\right|$ is plotted as a function of $x_{0} / \Lambda$ for $\Delta=1 / 2\left(l_{x}=0.5 \Lambda\right), A / a=0.3$ and $a / \Lambda=0.2$. According to Eq. (14), the point of unstable equilibrium is $\tilde{x}_{0}=5 \Lambda / 8$, i.e. it is asymmetric being shifted from the middle of a period. It is seen also that the magnitudes of maximum and minimum values of the lateral Casimir force in this case differ by 1.9. Needless to say, the work done by the lateral force over one period is equal to zero as it should be in any adiabatic process. If $\Delta$ is further increased, the position of the points of unstable equilibrium will be even more shifted to the right boundary of the period with an increase of the ratio of magnitudes of the maximum to minimum lateral forces.

In the above the method of pairwise summation and perturbation theory in relative distortion amplitudes were applied to obtain the general expression for the lateral Casimir force between metallic plates with longitudinal corrugations of arbitrary profile. The pairwise summation works well when the corrugation period is several times larger than the separation between plates. The obtained expressions were used in the case of asymmetric saw tooth 
like structures which lack the right-left symmetry of sinusoidal corrugations. It was shown that with the proper choice of the corrugation shape and parameters, it is possible to change not only the magnitude of the lateral Casimir force, but make it asymmetric and affect the character of points of equilibrium. In particular, the maximum to minimum force ratio can be increased by several times. This opens new opportunities to control the lateral Casimir force for the diversified applications in microelectromechanical devices based entirely on the vacuum fluctuation properties of quantum electrodynamics.

This work was supported by the National Institute of Standards and Technology, through a Precision Measurement Grant and the University of California and Los Alamos National Laboratory through the LANL-CARE program. G.L.K. and V.M.M. were also partially supported by CNPq and Finep (Brazil).

[1] H. B. G. Casimir, Proc. K. Ned. Akad. Wet. 51, 793 (1948).

[2] P. W. Milonni, The Quantum Vacuum (Academic Press, San Diego, 1994).

[3] V. M. Mostepanenko and N. N. Trunov, The Casimir Effect and its Applications (Clarendon Press, Oxford, 1997).

[4] K. A. Milton, The Casimir Effect (World Scientific, Singapore, 2001).

[5] M. Kardar and R. Golestanian, Rev. Mod. Phys. 71, 1233 (1999).

[6] M. Bordag, U. Mohideen, and V. M. Mostepanenko, Phys. Rep. 353, 1 (2001).

[7] S. K. Lamoreaux, Phys. Rev. Lett. 78, 5 (1997).

[8] U. Mohideen and A. Roy, Phys. Rev. Lett. 81, 4549 (1998); G. L. Klimchitskaya, A. Roy, U. Mohideen, and V. M. Mostepanenko, Phys. Rev. A 60, 3487 (1999).

[9] A. Roy and U. Mohideen, Phys. Rev. Lett. 82, 4380 (1999).

[10] A. Roy, C.-Y. Lin, and U. Mohideen, Phys. Rev. D 60, 111101(R) (1999).

[11] B. W. Harris, F. Chen, and U. Mohideen, Phys. Rev. A 62, 052109 (2000).

[12] T. Ederth, Phys. Rev. A 62, 062104 (2000).

[13] G. Bressi, G. Carugno, R. Onofrio, and G. Ruoso, Phys. Rev. Lett. 88, 041804 (2002).

[14] R. S. Decca, D. López, E. Fischbach, and D. E. Krause, Phys. Rev. Lett. 91, 050402 (2003).

[15] R. S. Decca, E. Fischbach, G. L. Klimchitskaya, D. E. Krause, D. López, and V. M. Mostepanenko, Phys. Rev. D 68, 116003 (2003). 
[16] M. Bordag, B. Geyer, G. L. Klimchitskaya, and V. M. Mostepanenko, Phys. Rev. D 58, 075003 (1998); 60, 055004 (1999); 62, 011701(R) (2000).

[17] V. M. Mostepanenko and M. Novello, Phys. Rev. D 63, 115003 (2001).

[18] E. Fischbach, D. E. Krause, V. M. Mostepanenko, and M. Novello, Phys. Rev. D 64, 075010 (2001).

[19] G. L. Klimchitskaya and U. Mohideen, Int. J. Mod. Phys. A 17, 4143 (2002).

[20] H. B. Chan, V. A. Aksyuk, R. N. Kleiman, D. J. Bishop, and F. Capasso, Science 291, 1941 (2001); Phys. Rev. Lett. 87, 211801 (2001).

[21] E. Buks and M. L. Roukes, Phys. Rev. B 63, 033402 (2001).

[22] Yu. S. Barash, Izv. Vuzov. Ser. Radiofiz. 16, 1086 (1973) [Sov. Radiophys. 16, 945 (1973)].

[23] S. J. van Enk, Phys. Rev. A 52, 2569 (1995).

[24] R. Golestanian and M. Kardar, Phys. Rev. Lett. 78, 3421 (1997); Phys. Rev. A 58, 1713 (1998).

[25] T. Emig, A. Hanke, R. Golestanian, and M. Kardar, Phys. Rev. Lett. 87, 260402 (2001); Phys. Rev. A 67, 022114 (2003).

[26] F. Chen, U. Mohideen, G. L. Klimchitskaya, and V. M. Mostepanenko, Phys. Rev. Lett. 88, 101801 (2002); Phys. Rev. A 66, 032113 (2002).

[27] M. Bordag, G. L. Klimchitskaya, and V. M. Mostepanenko, Int. J. Mod. Phys. A 10, 2661 (1995).

[28] C. Genet, A. Lambrecht, P. Maia Neto, and S. Reynaud, Europhys. Lett. 62, 484 (2003). 
Figures 


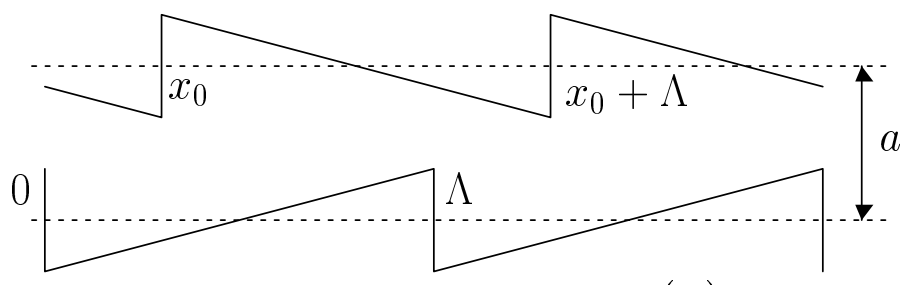

(a)

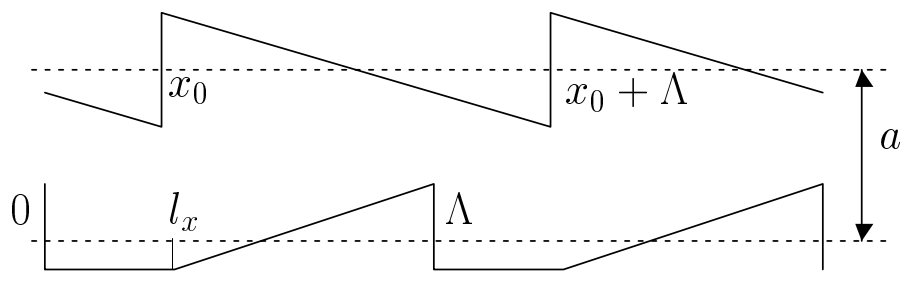

(b)

FIG. 1: The saw tooth corrugations on both plates with a phase shift $x_{0}$ and period $\Lambda$ (a). On lower plate the flat segments of length $l_{x}$ are added, while preserving the same corrugations on upper plate (b). 

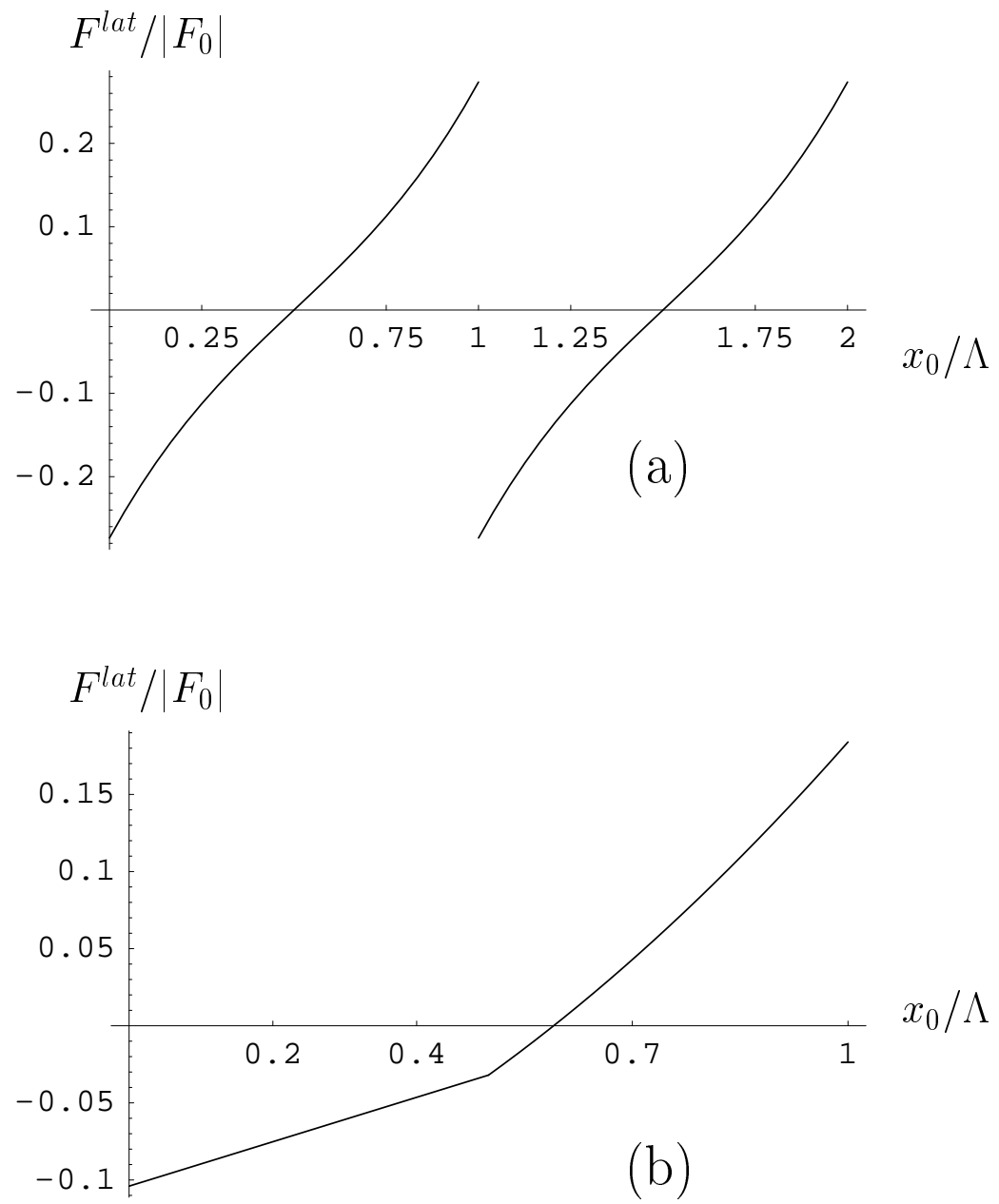

FIG. 2: The relative lateral Casimir force as a function of a relative phase shift for the corrugations of Fig. 1,a and Fig. 1,b [(a), (b), respectively]. 